\title{
Mixed Stimulus-Induced Mode Selection in Neural Activity Driven by High and Low Frequency Current under Electromagnetic Radiation
}

\author{
Lulu Lu, ${ }^{1}$ Ya Jia, ${ }^{1}$ Wangheng Liu, ${ }^{2}$ and Lijian Yang ${ }^{1}$ \\ ${ }^{1}$ Institute of Biophysics and Department of Physics, Central China Normal University, Wuhan 430079, China \\ ${ }^{2}$ School of Electrical and Electronic Engineering, Wuhan Polytechnic University, Wuhan 430023, China \\ Correspondence should be addressed to Ya Jia; jiay@mail.ccnu.edu.cn
}

Received 2 August 2017; Revised 15 September 2017; Accepted 27 September 2017; Published 19 October 2017

Academic Editor: Zeraoulia Elhadj

Copyright (C) 2017 Lulu Lu et al. This is an open access article distributed under the Creative Commons Attribution License, which permits unrestricted use, distribution, and reproduction in any medium, provided the original work is properly cited.

\begin{abstract}
The electrical activities of neurons are dependent on the complex electrophysiological condition in neuronal system, the threevariable Hindmarsh-Rose (HR) neuron model is improved to describe the dynamical behaviors of neuronal activities with electromagnetic induction being considered, and the mode transition of electrical activities in neuron is detected when external electromagnetic radiation is imposed on the neuron. In this paper, different types of electrical stimulus impended with a highlow frequency current are imposed on new HR neuron model, and mixed stimulus-induced mode selection in neural activity is discussed in detail. It is found that mode selection of electrical activities stimulated by high-low frequency current, which also changes the excitability of neuron, can be triggered owing to adding the Gaussian white noise. Meanwhile, the mode selection of the neuron electrical activity is much dependent on the amplitude $B$ of the high frequency current under the same noise intensity, and the high frequency response is selected preferentially by applying appropriate parameters and noise intensity. Our results provide insights into the transmission of complex signals in nerve system, which is valuable in engineering prospective applications such as information encoding.
\end{abstract}

\section{Introduction}

Dynamic behaviors of neurons are significant to acquaintance with signal exchange in the brain or even with related diseases; as a result, preceding works have been investigated [1-9]; based on this, several models are established to study mode selection of neurons. The external forcing current and other bifurcation parameters [10-26] are used to investigate the transition between modes of electrical activities and dynamical response because the excitability of neuron can be changed by external electric stimuli effectively. For example, Wang et al. $[10,11]$ studied the fact that neuron electrical activities can be modulated by an external electric field and the responses of three classes of Morris-Lecar neurons to sinusoidal inputs and synaptic pulse-like stimuli. Lv et al. [12] investigated mode transition of electrical activity in a neuron under magnetic flow effect. Wang et al. [13] discussed the possible electric response in biological neurons under electric stimulus. Ciszak and Bellesi [14] considered a network of coupled neurons in which the strength of the interactions is modulated by synaptic long term potentiation and depression. Tang et al. [15] confirmed that the membrane potential of a neuron depends on the changes of transmembrane current, opening/closing of ion channels, and even the regulation induced by astrocyte in a network composed of neuron bridged by astrocyte, and a review for dynamics in neuron and neuronal network $[16,17]$ was also provided for possible guide. Zhang et al. [18] suggested that the HR system may give an effective response to external stimulus by adjusting its intrinsic parameter, and this transition mode confirmed self-adaption of neuron. Djeundam et al. [19] analyzed the bifurcations occurring in the 3D HR model with and without random signal.

Indeed, the relevant topics about neurodynamics and neuronal network have been investigated widely by the known neuron models [20-29]. For example, Jun et al. 
[20] investigated dynamic characteristics of the fractionalorder Hindmarsh-Rose (HR) neuronal model, and the results showed possible differences in dynamic characteristic between the fractional-order HR neuronal model and an integer-ordered model. Guo et al. [21] found that both excitatory and electrical autapses increase the occurrence of burst firing, thus reducing neuronal firing regularity. It is also found that a time delay could decrease and suppress the chaotic state in the case of inhibitory chemical selfconnections with a proper autaptic intensity [22-24]. Yi et al. [25] constructed the fact that neuron can exhibit different spiking patterns, even chaotic behaviors, depending on the values of stimulus frequencies. Baptista et al. [27] studied the combined action of chemical and electrical synapses in small networks of HR neurons.

It is thought that real neuron activities are complex and the oscillatory behaviors depend on the energy supply; as a result, the calculation and estimation of general energy are very important in dynamical systems. For example, the Hamilton energy function [28] of generic dynamical system is investigated to observe the emergence of action potential and mode transition in electrical activities. Yamada and Kashimori [29] found that the learning of face stimuli makes the functional connections between these inferior temporal networks. Ma et al. [30-32] investigated the death and breakup of spiral wave in the neuronal network. With the deepening of research development, more and more achievements are observed [33-37]. Pinto et al. [33] analyzed transitions between synchrony and asynchrony in both slow oscillations and fast spikes by adding artificial electrical coupling. Gu et al. [34] confirmed the on-off firing pattern as noise-induced stochastic firing pattern near a subcritical Hopf bifurcation point. Yilmaz et al. [36, 37] explained the effects of an autapse localized pacemaker activity across a Newman-Watts small-world network consisting of stochastic Hodgkin-Huxley neurons.

As it can be known, the relevant experiments of the neuron electrical activity play a vital role in achieving biological functions of the nervous system [38-43]. The latest researches described the complex bifurcations related to real experiments and HR neuron model; the experimental results $[38,39]$ demonstrate the dynamics of a neuronal transition from chaotic bursting to chaotic spiking in the nervous system, which agree with published findings in theoretical neuronal models [40,41]. In addition, it is found that the firing, spike frequency, and instantaneous spike frequency observed in the experiment were simulated and explained using HH models. References $[42,43]$ illustrate the dynamics of different firing patterns and the frequency and temporal coding mechanisms of aortic baroreceptor.

Neuron can be thought as an effective signal processor and different external stimuli are encoded to give possible outputs and response; thus signal can be propagated in the nerve systems. In most of the previous works, specific external stimulus is often used. In fact, neurons can receive many signal inputs synchronously via multiple channels; as mentioned in [44], a low frequency (LF) signal and a high frequency (HF) forcing simultaneously confirmed that neuron gave response sensitively to intensity compared with angular frequency completely, and the energy dependence on external forcing was also discussed. The potential mechanism is that stronger stimulus can input enough energy to induce mode transition while angular frequency can cause slight modulation on firing rhythm at fixed intensity. Besides the mode selection and transition in electrical activities, coherence resonance is also interesting by applying appropriate forcing and changing membrane temperature [45, 46]. Electromagnetic induction and radiation are important phenomenon in excitable media because external electromagnetic field can cause polarization and magnetization in molecular level. Up to now, many nonlinear electric devices such as negative resistor and memristor [47-49] can be used to design oscillatory circuit and some circuits could be effective in reproducing electrical activities in neurons. But, the most important aspect could be that potential mechanism and application should be considered in physical view. For example, electromagnetic induction on neuron generates induction current and excitability of neuron is changed [50], and external electromagnetic radiation could further induce multiple mode response in electrical activities of neuron.

The high-low frequency signal is wildly applied in the nonlinear system, which amount to two periodic forces. Such two-frequency periodic forces are often used in many different fields, including commutation technologies, where information carriers are usually HF signal modulated by LF signal that encodes the data. The application of high-low frequency signals is pervasive in neural systems; for example, bursting neurons exhibit two widely different time scales; two frequency signals correspond to different signal input channels in the neuron. In addition, HF stimulation is playing an important role in biological phenomena, such as increased drug uptake by brain cells, resonantly enhanced biodegradation of microorganisms, and ultrasonic irradiation of two different frequency signals which has been seen to enhance cavitation yield.

In this paper, different types of electrical stimulus impended with a high-low frequency current are imposed on HR neuron model, and the mixed stimulus-induced mode selection in neural activity is discussed; thus the excitability of neuron is time-varying. It is found that the mode transition in electrical activity can be investigated by adding the Gaussian white noise. Meanwhile, it might be significant to explain the phenomenon of signal transmission in neurons when other signal types are imposed simultaneously.

\section{Model and Scheme}

According to the Maxwell electromagnetic induction theorem, the influence of dynamic behavior on the electrical activity of each neuron can be attributed to the bioelectricity within the nervous system (e.g., fluctuation of ion concentration between the inside and outside of the cell). Therefore, in this improved HR neuron model [50], the fluctuation of electromagnetic radiation is established, and the influence of magnetic flux is considered during the emergence of collective electrical activities and signals propagation among 
a large set of neurons. The dynamical equations for the new neuron model are described as follows:

$$
\begin{aligned}
& \frac{d x}{d t}=y-a x^{3}+b x^{2}-z-k_{1} \rho(\varphi) x+I_{\mathrm{ext}}, \\
& \frac{d y}{d t}=c-d x^{2}-y, \\
& \frac{d z}{d t}=r[s(x+1.6)-z], \\
& \frac{d \varphi}{d t}=k x-k_{2} \varphi,
\end{aligned}
$$

where $x, y, z$, and $\varphi$ describe the membrane potential, the slow current associated with recovery variable, the adaption current, and the magnetic flux across the membrane of neuron, respectively. The parameters are selected as $a=1, b=$ $3, c=1, d=5, r=0.006$, and $s=4$. The external forcing current $I_{\text {ext }}$ is the direct current when $I_{\text {ext }}=I$, and $I_{\text {ext }}$ is the high-low frequency current when $I_{\text {ext }}=I+A \cos (\omega t)+$ $B \cos (N \omega t) . k x$ and $k_{2} \varphi$ describe the membrane potentialinduced changes on magnet flux and the leakage of magnet flux, respectively. The term $k_{1} \rho(\varphi) x$ is the feedback current on the membrane potential induced by electromagnetic induction, where $k_{1}$ is the feedback gain. The physical unit is verified as follows:

$$
i^{\prime}=\frac{d q(\varphi)}{d t}=\frac{d q(\varphi)}{d \varphi} \frac{d \varphi}{d t}=\rho(\varphi) V=k_{1} \rho(\varphi) x
$$

where $V$ is the induced voltage and has the same physical units as variable $x$, and the dependence of electric charge on magnet flux defined by the memory conductance is

$$
\rho(\varphi)=\frac{d q(\varphi)}{d t}=\alpha+3 \beta \varphi^{2}
$$

where $\alpha, \beta$ are parameters and $q$ is the charge across the memristor. The $\rho(\varphi)$ is the memory conductance of a magnetic flux-controlled memristor $[47,48]$, which is used to describe the coupling between magnetic flux and membrane potential of neurons. In fact, induction current can be triggered and fed back to membrane potential via the memristor coupling, and the excitability of neuron can be decreased. With this improved model, many phenomena of electrical activity in the neuron are investigated under the electromagnetic induction. Compared with the previous models, our new model holds more variable parameters and presents more complex dynamical behaviors by analyzing the bifurcation of interspike interval (ISI).

\section{Results and Discussion}

In the numerical studies, the parameters are selected as $k=$ $0.9, k_{1}=0.4, k_{2}=0.5, \alpha=0.4$, and $\beta=0.02$, the fourth-order Runge-Kutta algorithm is used, the time step is set as 0.001 , the transient period for calculating is 1500 time units, and the initial values for $(x, y, z, \varphi)$ are set as $(0.1,0.2,0.1,0)$.

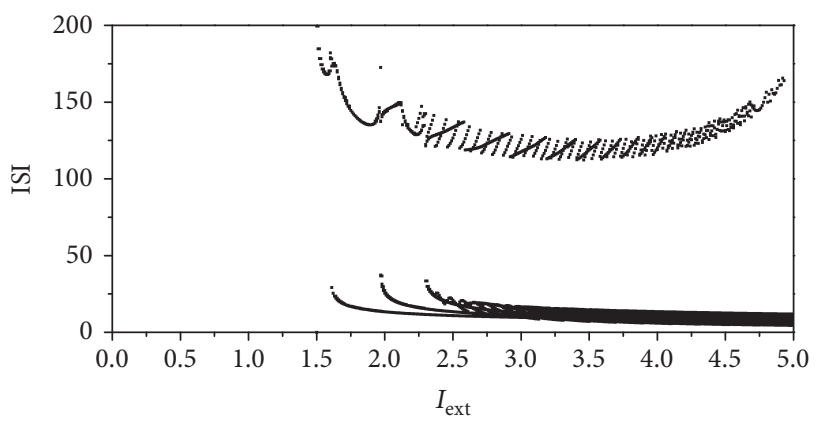

FIgURE 1: Bifurcation diagram with the increasing of external direct current $I_{\text {ext }}$.

3.1. Neuron Electrical Activities Stimulated by External Direct Current. In statistical view, the interspike interval is a measure of the order in electrical activity of neuron. The bifurcation diagram of ISI (i.e., the interspike interval) is plotted with the increasing of external direct current $I_{\text {ext }}=I$, and numerical results are presented by Figure 1.

Although the excitability and response in electrical activities are changed by induction current equation (2), Figure 1 shows that the interspike interval of neuron is not detected since the electric activity of neuron is quiescent state when the external direct current $I_{\text {ext }}$ is increased from 0 to 1.5. However, it is also shown that there are multiple modes in electrical activities of neuron when the external direct current $I_{\text {ext }}$ is increased from 1.5 to 5.0 .

Under different external direct current $I_{\text {ext }}$, the sampled time series of electrical activities of neuron are shown by Figure 2, which can explore the dynamical properties of electrical activities of neuron. It is also found that the quiescent, spiking, bursting, and periodical states can be observed by selecting appropriate external direct current $I_{\text {ext }}$.

3.2. Neuron Electrical Activities Stimulated by High-Low Frequency Current. When the periodical type of external highlow frequency current $I_{\text {ext }}=I+A \cos (\omega t)+B \cos (N \omega t)$ is considered, for simplicity, we set $I=0$; the term $A \cos (\omega t)$ is external low frequency (LF) signal with amplitude $A$, and the term $B \cos (N \omega t)$ is high frequency (HF) modulation with amplitude $B$, whose angular frequency is $N$ times that of the low frequency signal, $N \gg 1$. Then the change of bifurcation diagram of ISI is calculated with different parameters, and the results are shown in Figure 3.

Figure 3 confirmed that the parameters of external highlow frequency current (i.e., $B, \omega, N$ ) play a crucial role in the varieties of electrical activities when electromagnetic induction $\varphi$ is considered. Comparing the ISI diagram of the three different parameters, the mode in electrical activities of neuron is much dependent on the parameter $\omega$; this underlying mechanism might be that the low frequency signal is more important. Figure 3(a) shows that appropriate large amplitude $B$ is effective in making the electrical activities appear in the continuous bursting state. It is found that the bursting electrical activities can be transformed into spiking state and quiescent state (when $\omega$ value ranges from 0.17 to 0.2 , the ISI value is zero) by increasing the parameter $\omega$ value as shown 


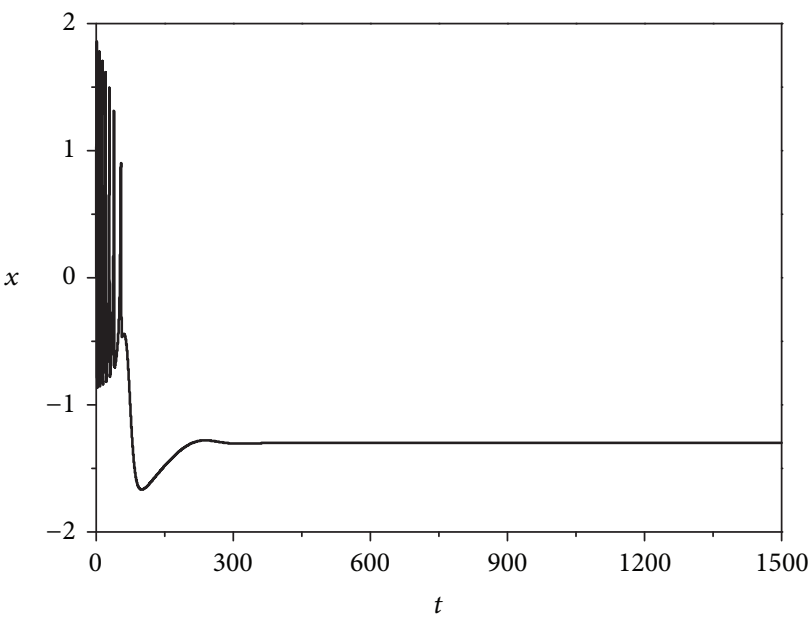

(a)

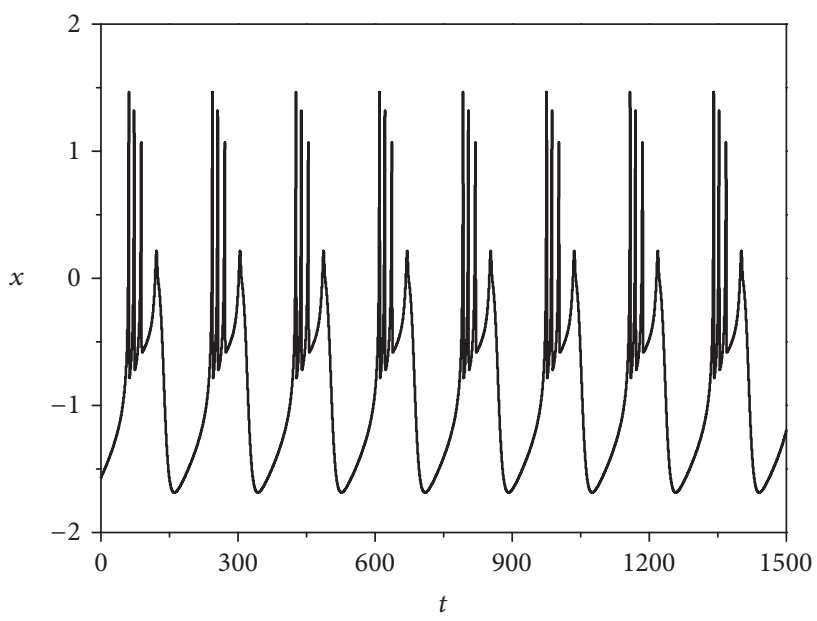

(c)

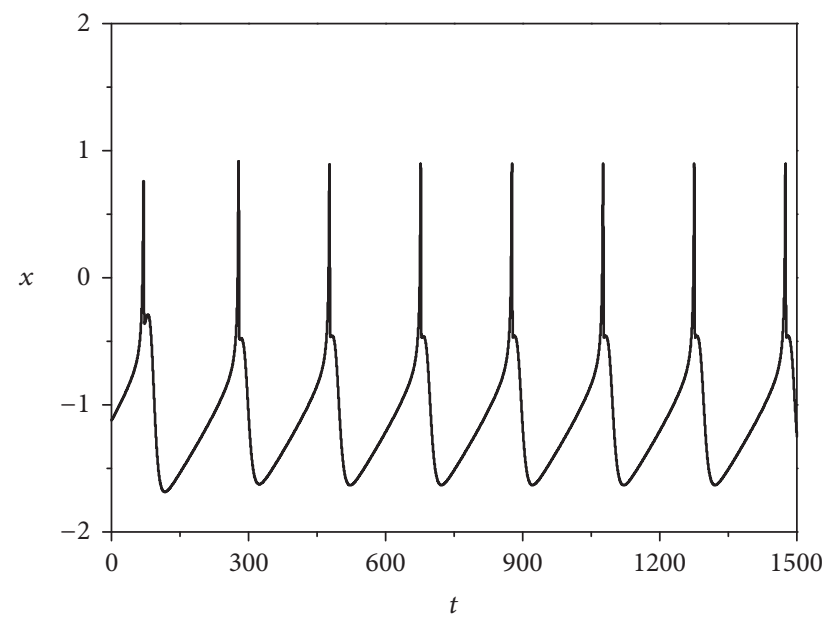

(b)

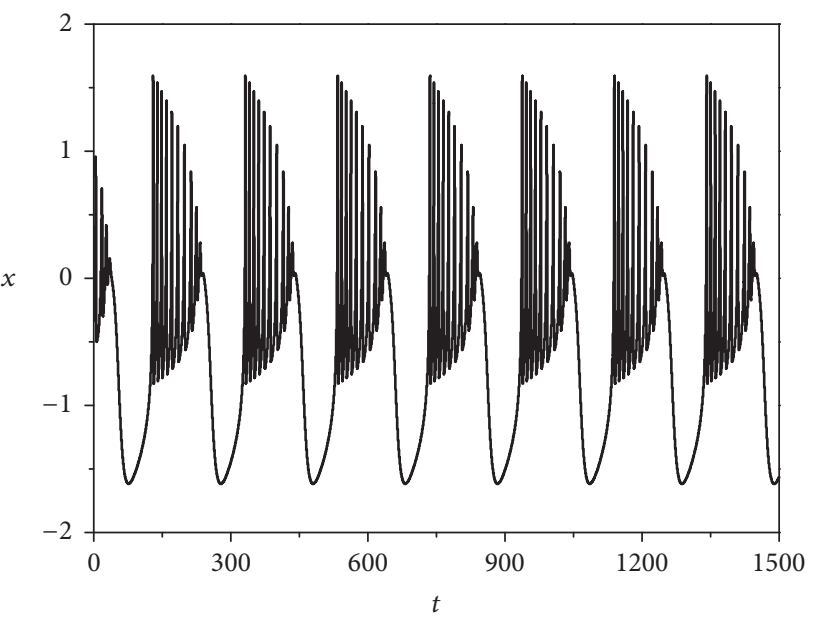

(d)

FiguRE 2: Sampled time series of membrane potential under different external direct current $I_{\text {ext }}$. (a) $I_{\text {ext }}=1.0$. (b) $I_{\text {ext }}=1.5$. (c) $I_{\text {ext }}=2.3$. (d) $I_{\text {ext }}=3.5$.

in Figure 3(b). The mode transformation by changing the parameter $N$ in neuron also can be observed in Figure 3(c).

With the increasing of amplitude value $B$ of $\mathrm{HF}$, Figures 4(a)-4(d) show that the electrical activities of neuron can transform the spiking state into the double bursting state. With the increasing of frequency $\omega$, Figures $4(\mathrm{e})-4(\mathrm{~h})$ make it apparent that, for small values of the high-low frequency $\omega$, the electrical activity of the neuron is found in a spiking state. After increasing the frequency $\omega$ of the high-low frequency current beyond some threshold value, the mode in electrical activities of neuron undergoes a transition from the spiking state to the bursting one. However, if we further increase $\omega$, the electrical activity of neuron goes back to the spiking state. That is, the mode in electrical activities of neuron undergoes a succession of two transitions (spiking state $\rightarrow$ bursting state $\rightarrow$ spiking state). In addition, with the increasing of the parameter $N$ of HF, Figures 4(i)-4(l) confirm that the electrical activity has not been changed and the bursting state is maintained. This is consistent with the bifurcation diagram for ISI in Figure 3.
3.3. Electrical Activities Stimulated by Direct Current and Gaussian White Noise. It is obviously known that noise can change the dynamical properties in electrical activities of neurons; therefore, noise is imposed on the neuron to detect the possible mode transition in electrical activities; here we insert the noise into the electromagnet flux term of system (1); it reads

$$
\begin{aligned}
& \frac{d x}{d t}=y-a x^{3}+b x^{2}-z+I_{\mathrm{ext}}-k_{1} \rho(\varphi) x, \\
& \frac{d y}{d t}=c-d x^{2}-y, \\
& \frac{d z}{d t}=r[s(x+1.6)-z], \\
& \frac{d \varphi}{d t}=k x-k_{2} \varphi+\xi(t),
\end{aligned}
$$

where $\xi(t)$ is Gaussian white noise, which represents the effect of external field or electromagnetic radiation-induced 


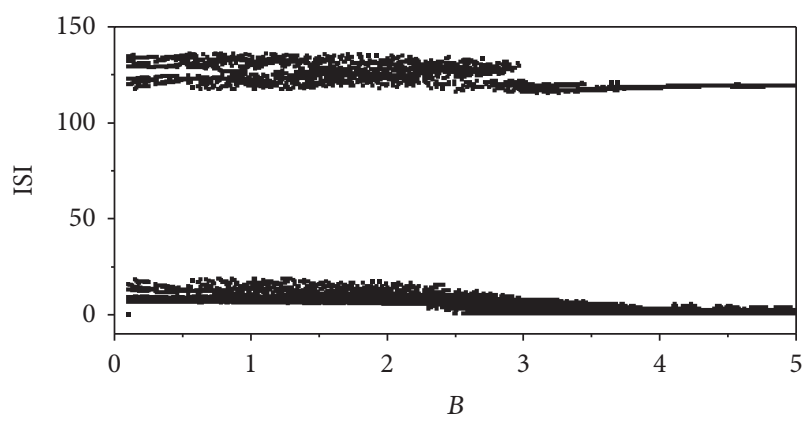

(a)

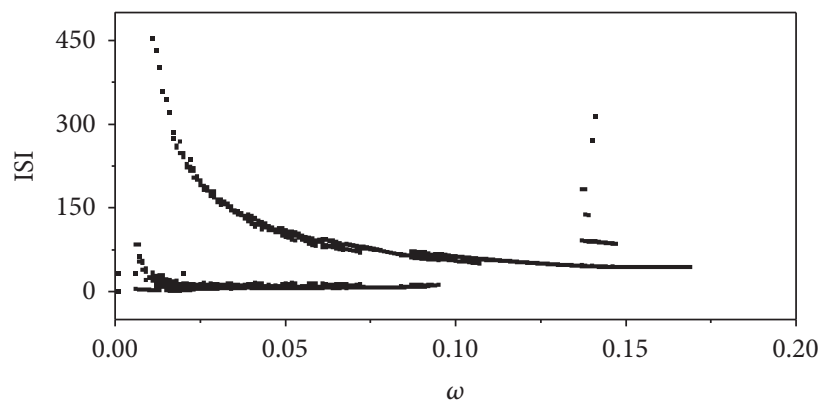

(b)

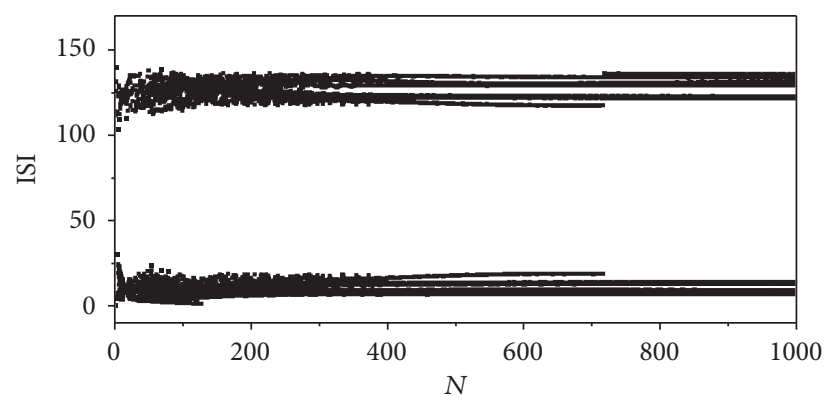

(c)

Figure 3: Bifurcation diagram with the increasing of different parameters. (a) $A=1.6, \omega=0.04$, and $N=200$. (b) $A=1.6, B=1.6$, and $N=200$. (c) $A=1.6, B=1.6$, and $\omega=0.04$.

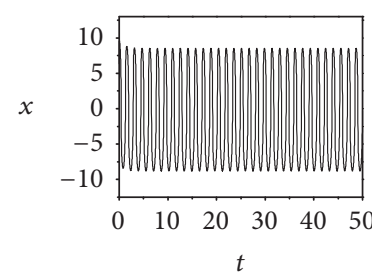

(a)

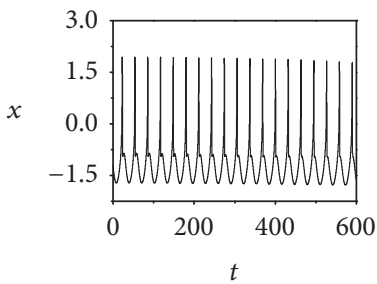

(e)

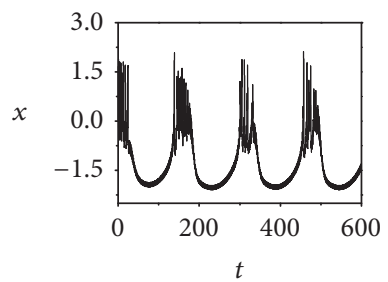

(i)

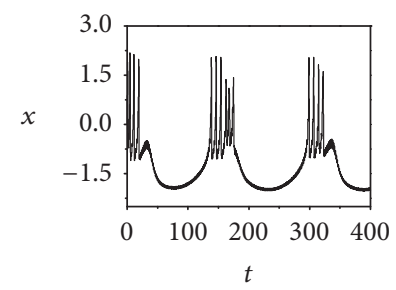

(b)

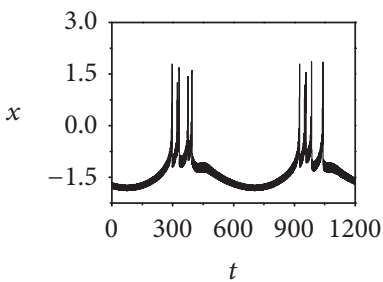

(f)

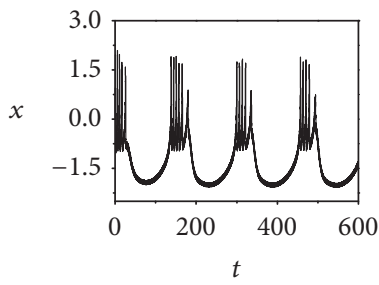

(j)

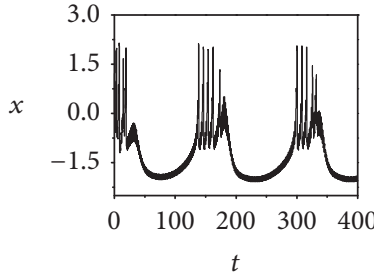

(c)

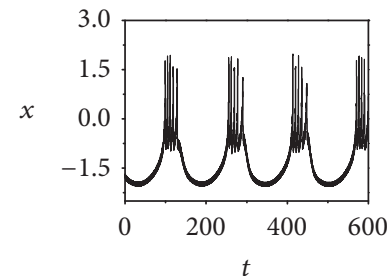

(g)

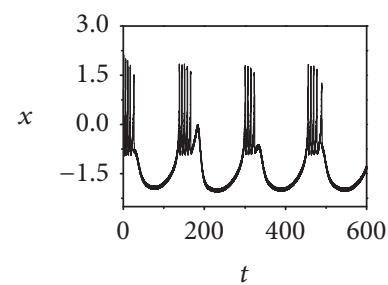

(k)

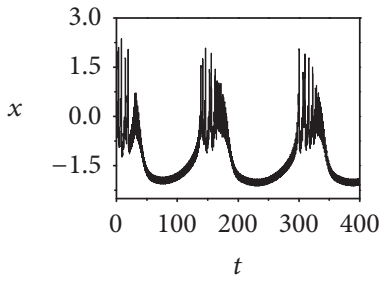

(d)

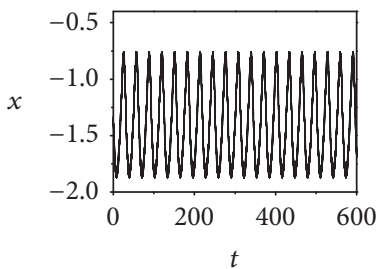

(h)

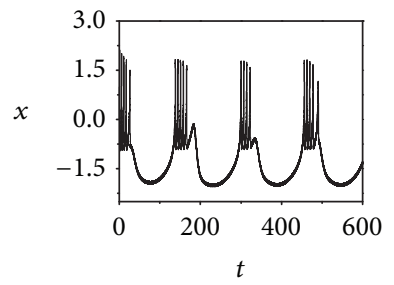

(l)

FIGURE 4: Sampled time series of membrane potential under different parameters. In the first layer, $A=1.6, \omega=0.04, N=200$, (a) $B=0.1$, (b) $B=0.9$, (c) $B=1.9$, and (d) $B=2.5$. In the second layer, $A=B=1.6, N=200$, (e) $\omega=0.001$, (f) $\omega=0.01$, (g) $\omega=0.04$, and (h) $\omega=0.2$. In the last layer, $A=B=1.6, \omega=0.04$, (i) $N=100$, (j) $N=300$, (k) $N=700$, and (l) $N=900$. The external high-low frequency current is set as $I_{\text {ext }}=A \cos (\omega t)+B \cos (N \omega t)$. 


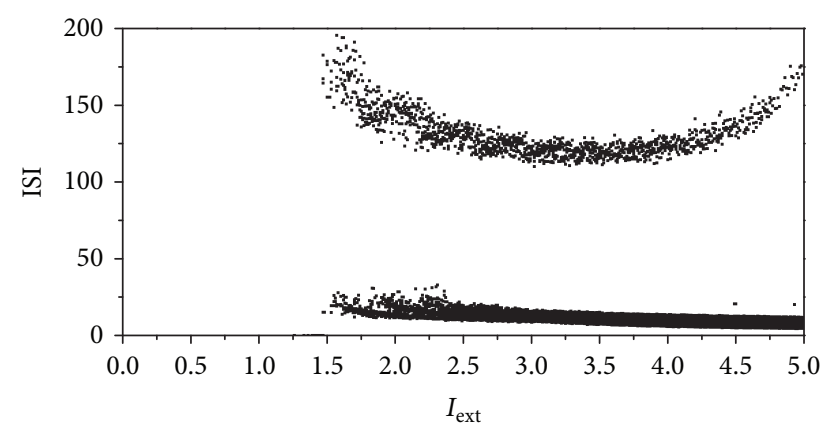

FIGURE 5: Bifurcation diagram with the increasing of external direct current $I_{\text {ext }} . D=0.2$.

magnet flux on the membrane; its statistical properties can be defined as $\langle\xi(t)\rangle=0,\left\langle\xi(t) \xi\left(t^{\prime}\right)\right\rangle=2 D \delta\left(t-t^{\prime}\right)$, and $D$ is the noise intensity.

When the external direct current $I_{\text {ext }}=I$ is considered, the bifurcation analysis is carried at fixed noise intensity $D=$ 0.2 of Gaussian white noise with the increasing of external direct current $I_{\text {ext }}$. Figure 5 shows that when the external direct current is in the range $0 \sim 1.4$, the electrical activity is in the quiescent state, and the interspike interval (ISI) value is zero. When the external direct current is in the range 1.4 5.0, the results of Figure 5 show that the mode of electrical activity of the neuron can be transformed and the bursting state can be reached when the small direct current is imposed under the Gaussian white noise.

The sample time series for membrane potential are calculated under different external direct currents in Figure 6. When the direct current is 1.40 , the electrical activity appears in the bursting state. Figures $6(a)-6(d)$ confirm that the different states (quiescent state, spiking state, and bursting state) can be observed by appending the Gaussian white noise.

\subsection{Electrical Activities Stimulated by High-Low Frequency} Current and Gaussian White Noise. When the periodical type of high-low frequency current $I_{\text {ext }}=I+A \cos (\omega t)+$ $B \cos (N \omega t)$ is considered (for simplicity, here we set $I=0$ ), Figure 7 shows the mode transformation of the neuron with the increase of the high-low frequency current parameters under the noise intensity $D=0.2$ of Gaussian white noise. In the first layer of panel in Figure 7(a), there are interspike intervals with the creasing of HF amplitudes, in the second layer in Figure 7(b), there is ISI with differential frequency of high-low frequency current, and in the third layer in Figure 7 (c), there is ISI with different parameter $N$ of the HF current.

Comparing the factors in Figure 7(a) with the distribution in Figure 3(a), it is interesting that they have a huge differentiation in the profile. Figures 7(a) and 3(a) show that a certain diversity of the neuronal electrical activity is observed when noise intensity is fixed, and the electrical activities can be effectively suppressed by increasing the amplitude $B$ of HF. The mode transition of the neuronal electrical activity is transformed from bursting state to spiking state and high frequency response of electrical activity is selected preferentially when the Gaussian white noise is imposed.

Comparing Figure $7(\mathrm{~b})$ with Figure 3(b), it is found that ISI of neuronal electrical activity become small with the frequency $\omega$ of high-low frequency current increases. Bursting state can be triggered and high frequency response of electrical activity is selected preferentially when high-low frequency current and the Gaussian white noise are imposed. Figures $7(\mathrm{c})$ and 3(c) show that the large transformations of electrical activities cannot be triggered with the increasing of HF current parameter $N$. Although the mode selection in neuron electrical activity is not much dependent on parameter $N$ of HF current, the mode in electrical activities of neuron is much dependent on the parameters (i.e., $B, \omega, N$ ). Furthermore, the relevant time series of membrane potential are provided with the increasing of different parameters (i.e., $B, \omega, N)$ in Figure 8.

It is confirmed that the bursting state of neuronal electrical activity can be changed into different mode by applying an appropriate intensity of the Gaussian white noise in Figures $8(\mathrm{a})-8(\mathrm{~d})$. With the increasing of the amplitude $B$ of HF current, the time series of electrical activity become more intensive. Figures $8(\mathrm{e})-8(\mathrm{~h})$ and $8(\mathrm{i})-8(\mathrm{l})$ show that the transformation of the neuronal electrical activity is not obvious with the increasing of the high frequency signal parameters when high-low frequency current and the Gaussian white noise are imposed; this is consistent with the bifurcation diagram for ISI in Figure 7.

Figure 9(a) is the time series of membrane potential when $A=1.6, \omega=0.04, N=200$, and $B=7.2$, and its magnification is shown in Figure 9(b). In order to observe the electrical activity of the neuron distinctly, the Fast Fourier Transform (FFT) is performed by the Origin software, and Fourier analysis converts a signal from its original domain (sampled time series) to the power spectrum in the frequency domain. The power spectra can be calculated by manipulating the FFT in Figure 9(c). We analyzed the results of intensive oscillations; the frequency of high peak distribution and low peak distribution (the frequency value is about 1.25 ) is between the external HF frequency value (about 0.04 ) and the external LF frequency value (about 8.0). It means that the electrical activity of the neuron is affected by the signal of high-low frequency current. Meanwhile, according to the frequency value of the above results, the mode selection in electrical activities of neuron might be more dependent on the low frequency current. Figure 9(c) shows that when the variation of power occurs around zero frequency, it signifies cluster discharge in neuron electrical activity, and when variation of power occurs around frequency 1.3, it illustrates that the electrical activities of neuron are of uniform period; this is consistent with the results in Figure 8.

Furthermore, the bifurcation diagrams are plotted with the increasing of the noise intensity $D$ in Figure 10. It can be seen that the mode transformation of the neuron electrical activity by changing amplitude $B$ of $\mathrm{HF}$ current or the frequency $\omega$ of high-low frequency current is more obvious than that of changing parameter $N$ under the different noise intensities. The effect of noise intensity is not obvious when high-low frequency current and the Gaussian white noise are 


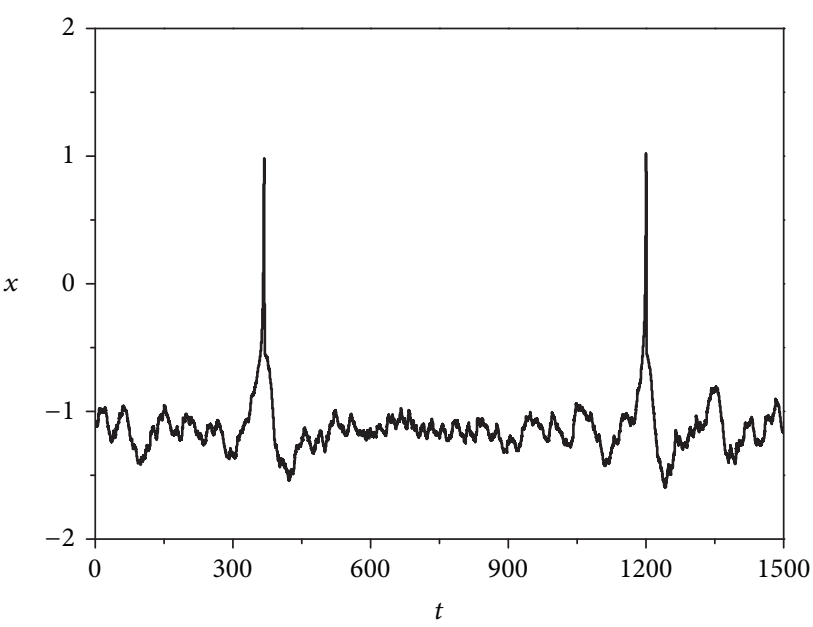

(a)

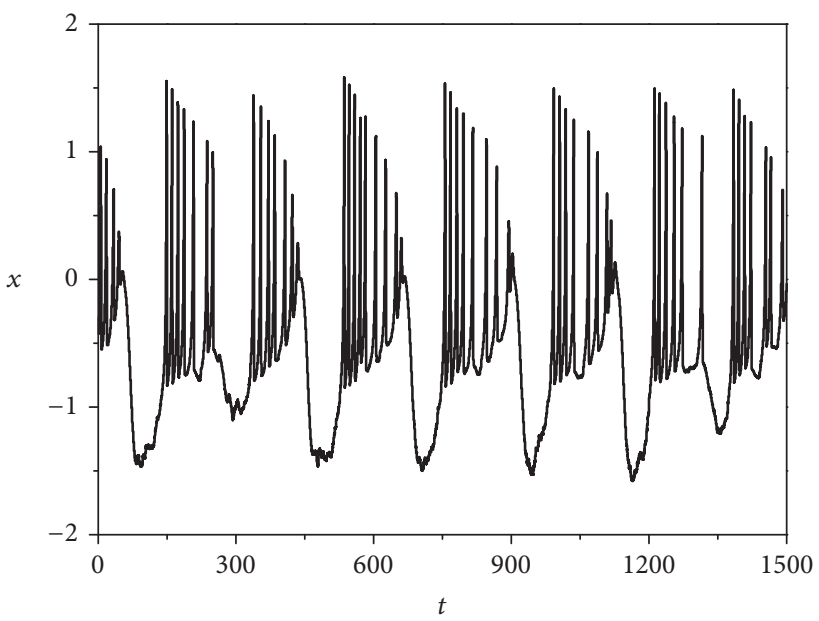

(c)

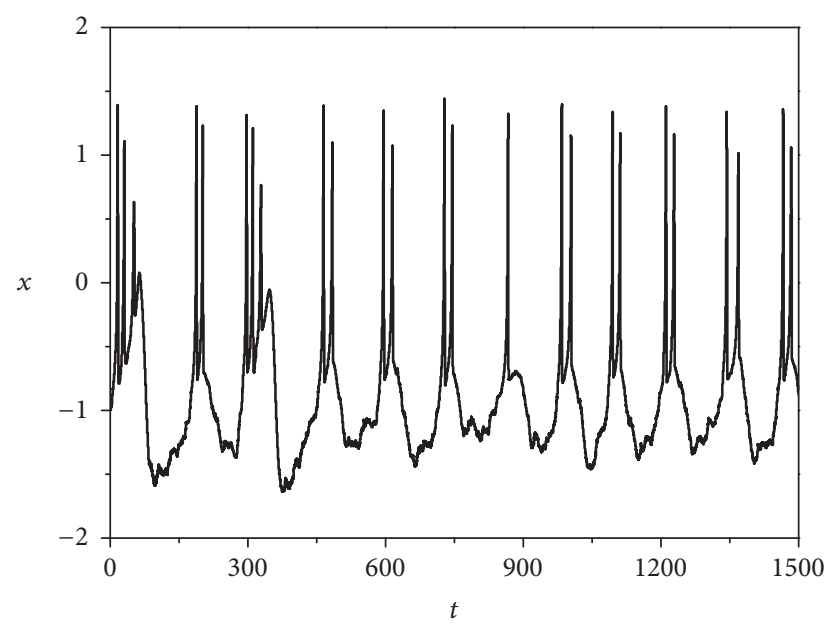

(b)

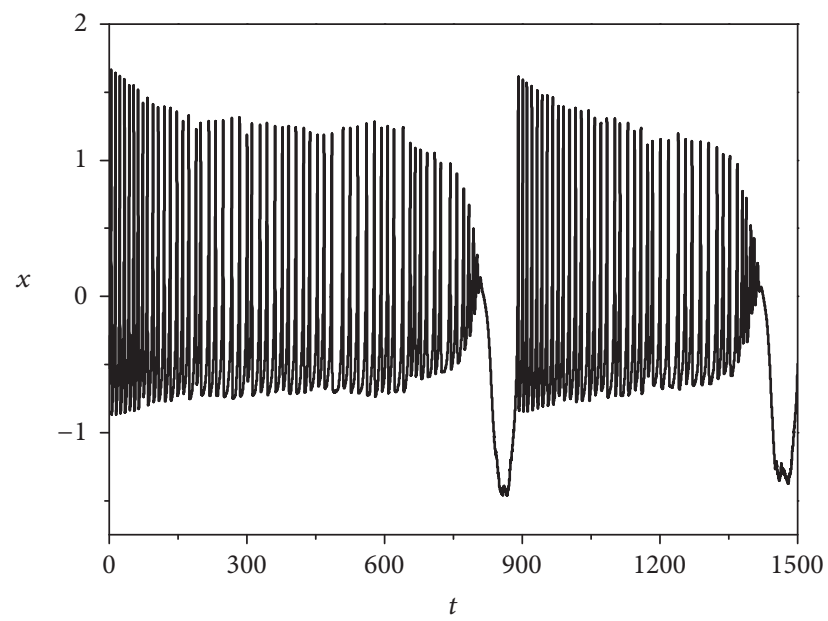

(d)

FIGURE 6: Sampled time series of membrane potential under different external direct current $I_{\text {ext }} . D=0.2$. (a) $I_{\text {ext }}=1.5$. (b) $I_{\text {ext }}=2.3$. (c) $I_{\text {ext }}=3.5$. (d) $I_{\text {ext }}=4.5$.

imposed. Figures 10(a) and 10(b) shows that, with increasing of amplitude $B$, the double bursting state of electrical activity is developed into the spiking state. However, as shown in both Figures $10(\mathrm{~b})$ and $10(\mathrm{c})$, by changing the frequency $\omega$, the double state of electrical activity is converted into the triple bursting state, and with alternation in parameter $N$ in Figures 10 (a) and $10(\mathrm{~d})$, the mode transformation of electrical activity is not obvious.

\section{Conclusions}

In this paper, different types of electrical stimulus impended with a high-low frequency current are imposed on new HR neuron model, and mixed stimulus-induced mode selection in neuron electrical activity is discussed in detail. Based on the improved HR neuron model, the dynamics of electrical activities of neuron is investigated by using the bifurcation analysis and the time series analysis. The effect of the highlow frequency current and the Gaussian white noise is also considered. It is found that the influence by the amplitude $B$ of the HF current in electrical activity of the neuron is larger than that of the other parameters (i.e., $\omega, N$ ) under the same noise intensity, and the high frequency response is selected preferentially by applying appropriate parameters and noise intensity. The underlying mechanism can be related to the induction current of high-low frequency signal.

\section{Open Problems}

In this previous investigations, mixed stimulus-induced mode selection in neuron electrical activity driven by highlow frequency current is discussed under the electromagnetic radiation. Therefore, it is interesting to investigate the phase shift between two periodic signals and the energy change between the different states in further works. Vibrational resonance within the whole parameter plane could be considered to get an insight into the transmission of LF signal in the excitable neuron system. In addition, the Fourier coefficient 


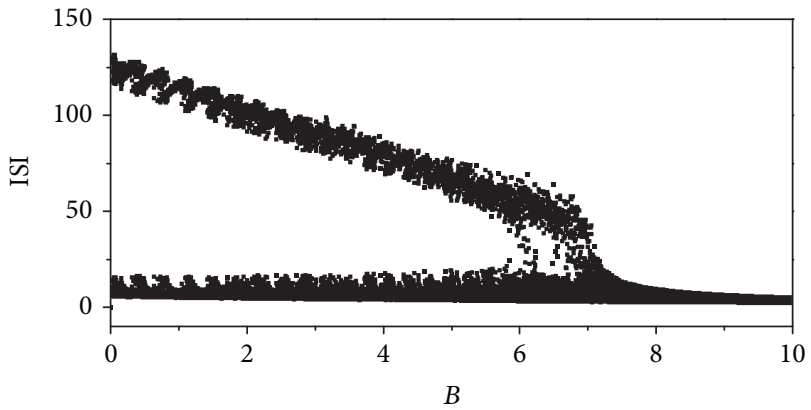

(a)

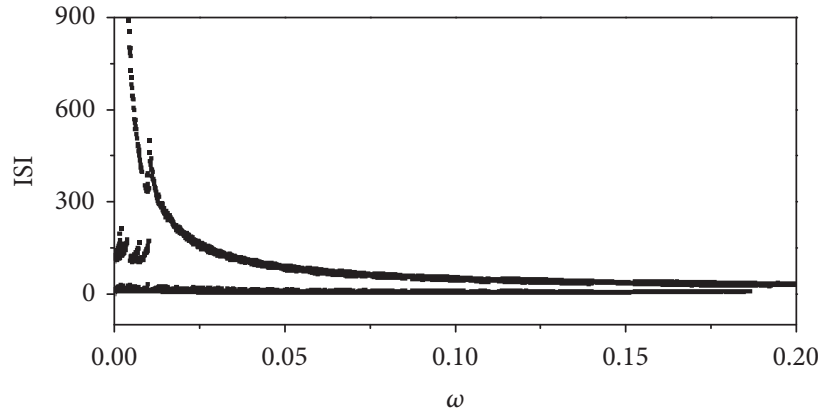

(b)

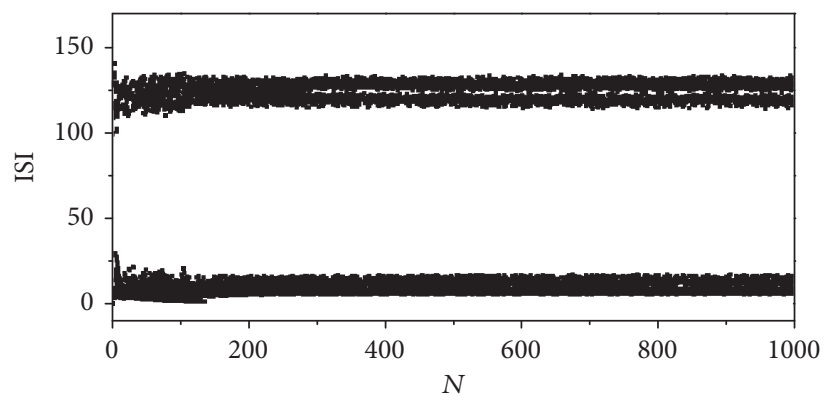

(c)

Figure 7: Bifurcation diagram with the increasing of different parameters. $D=0.2$. (a) $A=1.6, \omega=0.04$, and $N=200$. (b) $A=1.6, B=1.6$, and $N=200$. (c) $A=1.6, B=1.6$, and $\omega=0.04$.

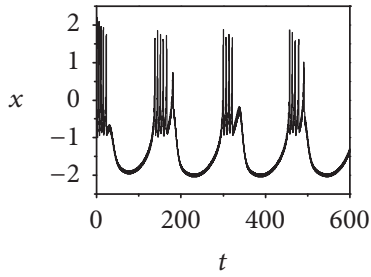

(a)

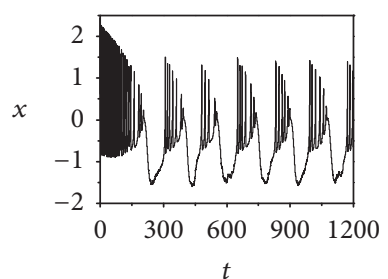

(e)

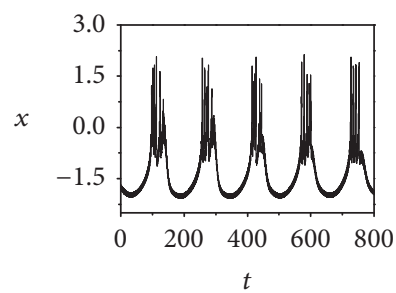

(i)

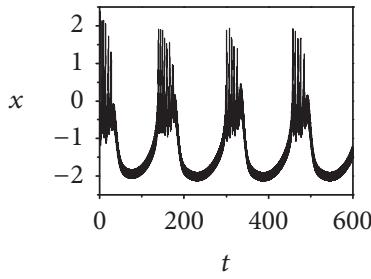

(b)

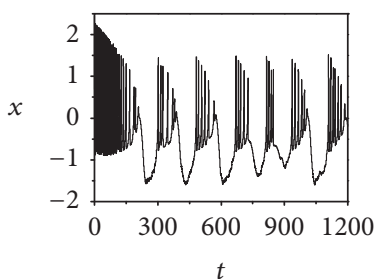

(f)

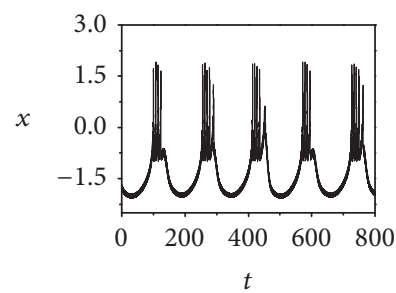

(j)

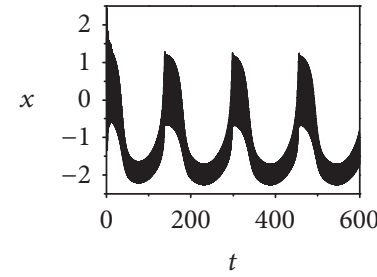

(c)

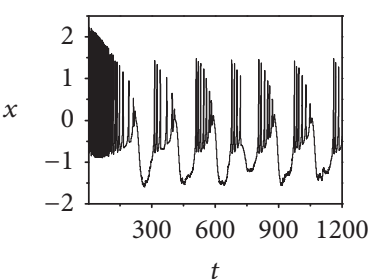

(g)

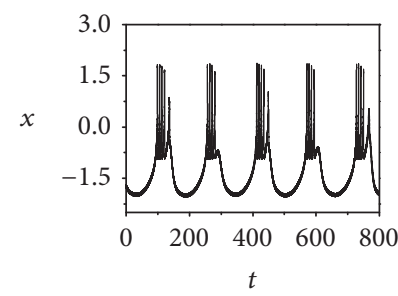

(k)

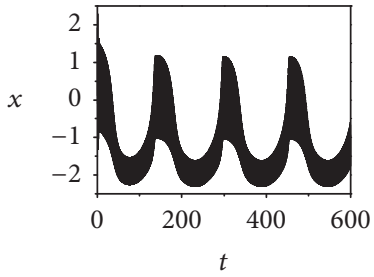

(d)

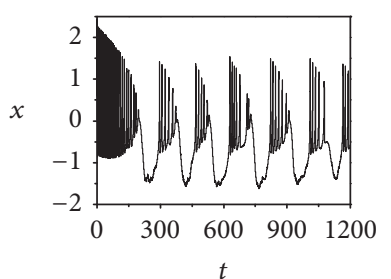

(h)

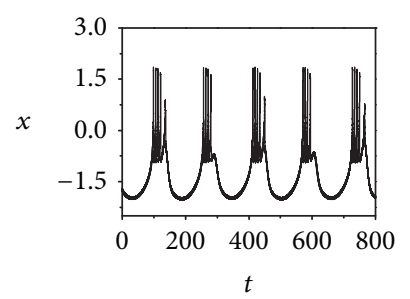

(1)

Figure 8: Sampled time series of membrane potential under different parameters. In the first layer, $A=1.6, \omega=0.04, N=200$, (a) $B=0.9$, (b) $B=3.0$, (c) $B=7.2$, and (d) $B=9.0$. In the second layer, $A=1.6, B=1.6, N=200$, (e) $\omega=0.001$, (f) $\omega=0.01$, (g) $\omega=0.04$, and (h) $\omega=0.2$. In the last layer, $A=1.6, B=1.6, \omega=0.04$, (i) $N=100,(\mathrm{j}) N=300$, (k) $N=700$, and (l) $N=900$. The Gaussian white noise intensity $D=0.2$, and the external high-low frequency current is set as $I_{\text {ext }}=A \cos (\omega t)+B \cos (N \omega t)$. 


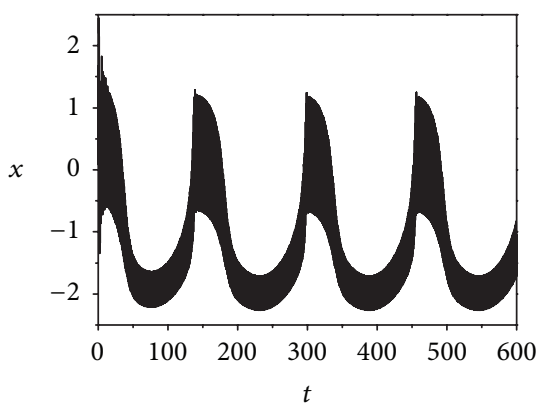

(a)

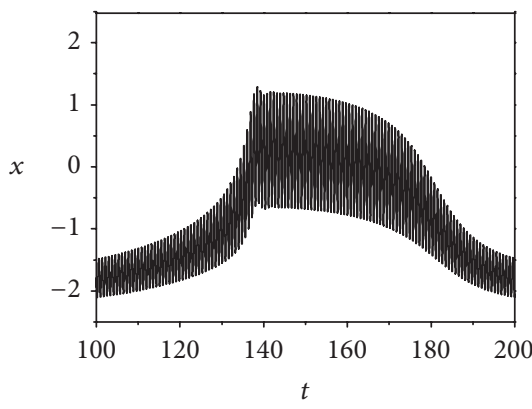

(b)

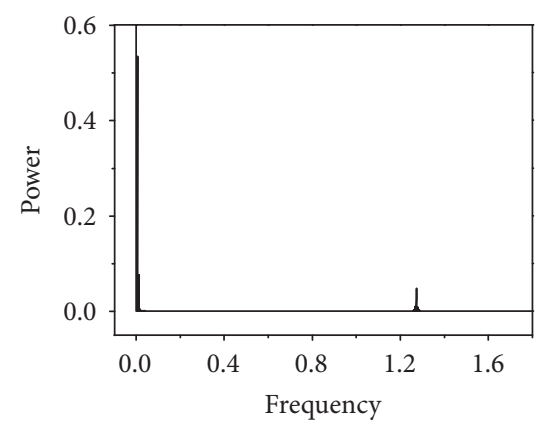

(c)

FIGURE 9: Sampled time series of membrane potential and power spectrum of electrical activity.

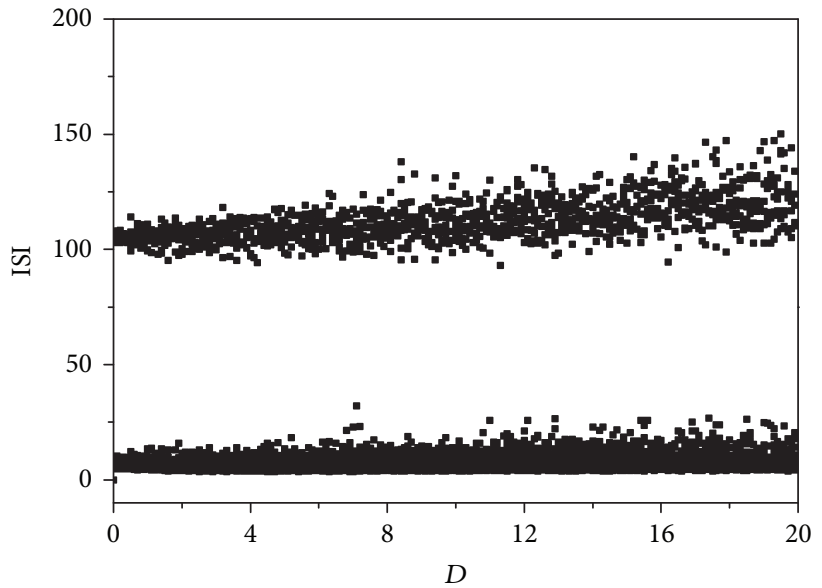

(a)

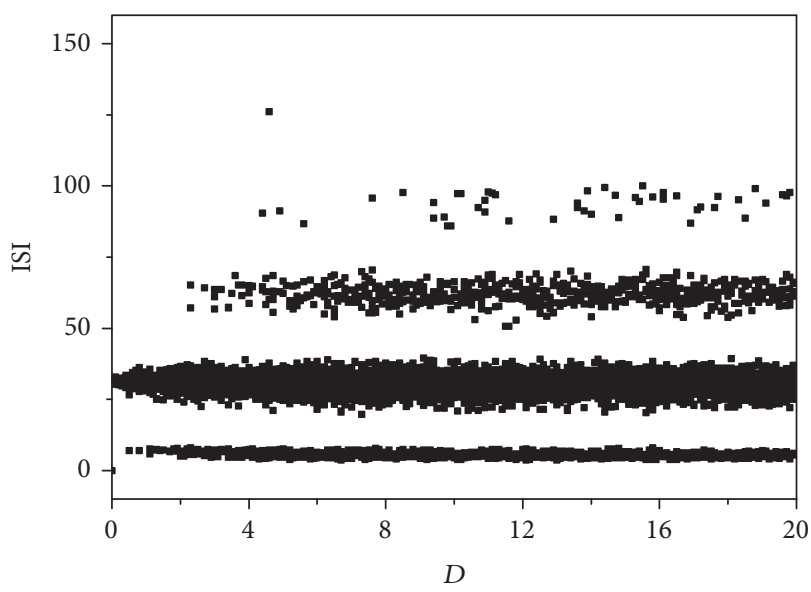

(c)

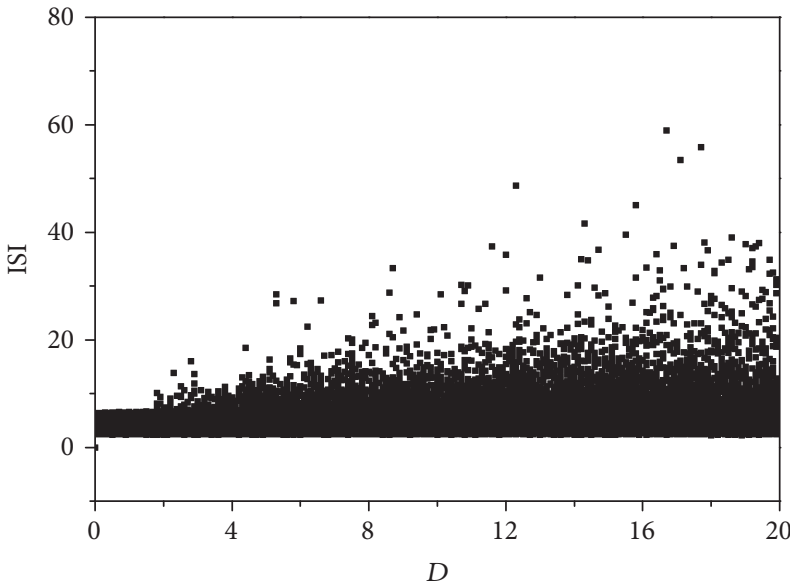

(b)

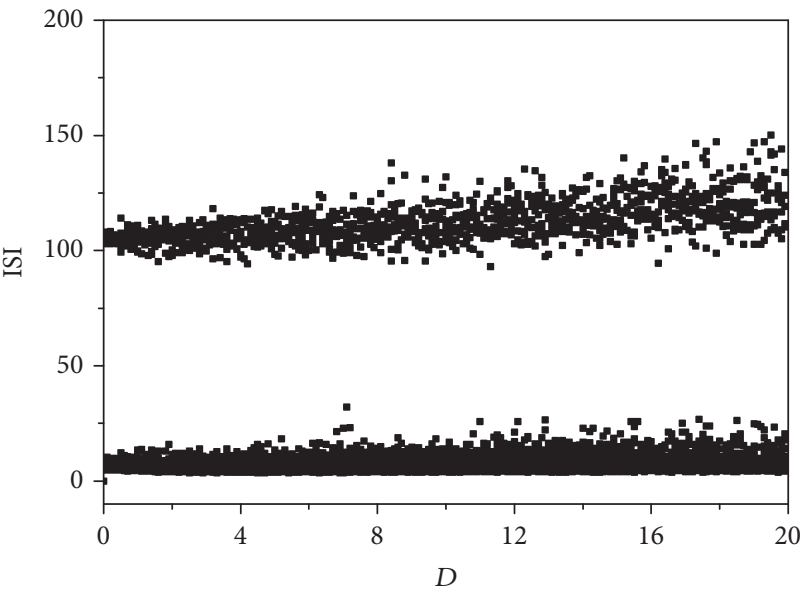

(d)

FIGURE 10: Bifurcation diagrams with the increasing of noise intensity $D$. (a) $A=1.6, B=1.6, \omega=0.04$, and $N=50$. (b) $A=1.6, B=9.0$, $\omega=0.04$, and $N=50$. (c) $A=1.6, B=1.6, \omega=0.2$, and $N=50$. (d) $A=1.6, B=1.6, \omega=0.04$, and $N=300$.

$Q$ and power spectrum could be introduced for evaluating the response of the system to the input LF signal. As it is known that the neuron network plays a significant role in the neuron system, therefore, the neuron network state transformation of different neuron models driving by the different external signals might be investigated under the electromagnetic field; the coherent resonance of the coupling neuron network is expected to be observed.

\section{Conflicts of Interest}

The authors declare that they have no conflicts of interest. 


\section{Acknowledgments}

The authors gratefully thank Professor Jun Ma from Lanzhou University of Technology for the constructive suggestions. This work was supported by the National Natural Science Foundation of China, under Grant nos. 11775091 and 11474117.

\section{References}

[1] A. L. Hodgkin and A. F. Huxley, "The dual effect of membrane potential on sodium conductance in the giant axon of Loligo," The Journal of Physiology, vol. 116, no. 4, pp. 497-506, 1952.

[2] R. Fitzhugh, "Impulses and physiological states in theoretical models," Biophysical Journal, vol. 1, pp. 445-466, 1961.

[3] G. B. Ermentrout and D. H. Terman, Mathematical Foundations of Neuroscience, Springer Science \& Business Media, New York, NY, USA, 2010.

[4] C. Morris and H. Lecar, "Voltage oscillations in the barnacle giant muscle fiber," Biophysical Journal, vol. 35, no. 1, pp. 193$213,1981$.

[5] J. L. Hindmarsh and R. M. Rose, "A model of the nerve impulse using two first-order differential equations," Nature, vol. 296, no. 5853, pp. 162-164, 1982.

[6] J. L. Hindmarsh and R. M. Rose, "A model of neuronal bursting using three coupled first order differential equations," Proceedings of the Royal Society of London B: Biological Sciences, vol. 221, no. 1222, pp. 87-102, 1984.

[7] T. R. Chay, "Chaos in a three-variable model of an excitable cell," Physica D: Nonlinear Phenomena, vol. 16, no. 2, pp. 233-242, 1985.

[8] L. Li, H. Gu, M. Yang, Z. Liu, and W. Ren, "A series of bifurcation scenarios in the firing pattern transitions in an experimental neural pacemaker," International Journal of Bifurcation and Chaos, vol. 14, no. 5, pp. 1813-1817, 2004.

[9] Z. Yang and Q. Lu, "Transitions from bursting to spiking due to depolarizing current in the Chay neuronal model," Communications in Nonlinear Science and Numerical Simulation, vol. 12, no. 3, pp. 357-365, 2007.

[10] H. Wang, L. Wang, L. Yu, and Y. Chen, "Response of MorrisLecar neurons to various stimuli," Physical Review E: Statistical, Nonlinear, and Soft Matter Physics, vol. 83, no. 2, Article ID 021915, 2011.

[11] H. Wang and Y. Chen, "Spatiotemporal activities of neural network exposed to external electric fields," Nonlinear Dynamics, vol. 85, no. 2, pp. 881-891, 2016.

[12] M. Lv, C. Wang, G. Ren, J. Ma, and X. Song, "Model of electrical activity in a neuron under magnetic flow effect," Nonlinear Dynamics, vol. 85, no. 3, pp. 1479-1490, 2016.

[13] H. Wang, Y. Chen, and Y. Chen, "First-spike latency in Hodgkin's three classes of neurons," Journal of Theoretical Biology, vol. 328, pp. 19-25, 2013.

[14] M. Ciszak and M. Bellesi, "Synaptic plasticity modulates autonomous transitions between waking and sleep states: Insights from a Morris-Lecar model," Chaos: An Interdisciplinary Journal of Nonlinear Science, vol. 21, no. 4, Article ID 043119, 2011.

[15] J. Tang, J.-M. Luo, and J. Ma, "Information transmission in a neuron-astrocyte coupled model," PLoS ONE, vol. 8, no. 11, Article ID e80324, 2013.

[16] J. Ma and J. Tang, "A review for dynamics in neuron and neuronal network," Nonlinear Dynamics, vol. 89, no. 3, pp. 15691578, 2017.
[17] J. Ma and J. Tang, "A review for dynamics of collective behaviors of network of neurons," Science China Technological Sciences, vol. 58, no. 12, pp. 2038-2045, 2015.

[18] J. Zhang, C. Wang, M. Wang, and S. Huang, "Firing patterns transition induced by system size in coupled Hindmarsh-Rose neural system," Neurocomputing, vol. 74, no. 17, pp. 2961-2966, 2011.

[19] S. R. D. Djeundam, R. Yamapi, T. C. Kofane, and M. A. AzizAlaoui, "Deterministic and stochastic bifurcations in the Hindmarsh-Rose neuronal model," Chaos, vol. 23, no. 3, p. 033125, 2013.

[20] D. Jun, Z. Guang-Jun, X. Yong, Y. Hong, and W. Jue, "Dynamic behavior analysis of fractional-order Hindmarsh-Rose neuronal model," Cognitive Neurodynamics, vol. 8, no. 2, pp. 167175, 2014.

[21] D. Guo, S. Wu, M. Chen et al., "Regulation of Irregular Neuronal Firing by Autaptic Transmission," Scientific Reports, vol. 6, Article ID 26096, 2016.

[22] J. Tang, J. Zhang, J. Ma, G. Y. Zhang, and X. Q. Yang, "Astrocyte calcium wave induces seizure-like behavior in neuron network," Science China Technological Sciences, vol. 60, pp. 1011-1018, 2016.

[23] J. Ma, X. Song, J. Tang, and C. Wang, "Wave emitting and propagation induced by autapse in a forward feedback neuronal network," Neurocomputing, vol. 167, pp. 378-389, 2015.

[24] H. Wang, J. Ma, Y. Chen, and Y. Chen, "Effect of an autapse on the firing pattern transition in a bursting neuron," Communications in Nonlinear Science and Numerical Simulation, vol. 19, no. 9, pp. 3242-3254, 2014.

[25] G.-S. Yi, J. Wang, C.-X. Han, B. Deng, and X.-L. Wei, "Spiking patterns of a minimal neuron to ELF sinusoidal electric field," Applied Mathematical Modelling, vol. 36, no. 8, pp. 3673-3684, 2012.

[26] Y. Li, G. Schmid, P. Hänggi, and L. Schimansky-Geier, "Spontaneous spiking in an autaptic Hodgkin-Huxley setup," Physical Review E: Statistical, Nonlinear, and Soft Matter Physics, vol. 82, no. 6, Article ID 061907, 2010.

[27] M. S. Baptista, F. M. Moukam Kakmeni, and C. Grebogi, "Combined effect of chemical and electrical synapses in HindmarshRose neural networks on synchronization and the rate of information," Physical Review E: Statistical, Nonlinear, and Soft Matter Physics, vol. 82, no. 3, Article ID 036203, 12 pages, 2010.

[28] C. N. Wang, Y. Wang, and J. Ma, "Calculation of Hamilton energy function of dynamical system by using Helmholtz theorem," Acta Physica Sinica, vol. 65, no. 24, Article ID 240501, 2016.

[29] Y. Yamada and Y. Kashimori, "Neural mechanism of dynamic responses of neurons in inferior temporal cortex in face perception," Cognitive Neurodynamics, vol. 7, no. 1, pp. 23-38, 2013.

[30] J. Ma, Y. Xu, G. Ren, and C. Wang, "Prediction for breakup of spiral wave in a regular neuronal network," Nonlinear Dynamics, vol. 84, no. 2, pp. 497-509, 2016.

[31] J. Ma, L. Huang, J. Tang, H.-P. Ying, and W.-Y. Jin, "Spiral wave death, breakup induced by ion channel poisoning on regular Hodgkin-Huxley neuronal networks," Communications in Nonlinear Science and Numerical Simulation, vol. 17, no. 11, pp. 4281-4293, 2012.

[32] J. Ma, L. Huang, H. P. Ying, and Z. S. Pu, "Detecting the breakup of spiral waves in small-world networks of neurons due to channel block," Chinese Science Bulletin, vol. 57, no. 17, pp. 20942101, 2012. 
[33] R. D. Pinto, P. Varona, A. R. Volkovskii, A. Szücs, H. D. Abarbanel, and M. I. Rabinovich, "Synchronous behavior of two coupled electronic neurons," Physical Review E: Statistical, Nonlinear, and Soft Matter Physics, vol. 62, no. 2, pp. 2644-2656, 2000.

[34] H. Gu, Z. Zhao, B. Jia, and S. Chen, "Dynamics of on-off neural firing patterns and stochastic effects near a sub-critical Hopf bifurcation," PLoS ONE, vol. 10, no. 4, Article ID e0121028, 2015.

[35] N. Korkmaz, İ. Öztürk, and R. Kılıç, "The investigation of chemical coupling in a HR neuron model with reconfigurable implementations," Nonlinear Dynamics, vol. 86, no. 3, pp. 18411854, 2016.

[36] E. Yilmaz, V. Baysal, M. Ozer, and M. Perc, "Autaptic pacemaker mediated propagation of weak rhythmic activity across smallworld neuronal networks," Physica A: Statistical Mechanics and its Applications, vol. 444, pp. 538-546, 2016.

[37] E. Yilmaz, M. Ozer, V. Baysal, and M. Perc, "Autapse-induced multiple coherence resonance in single neurons and neuronal networks," Scientific Reports, vol. 6, Article ID 30914, 2016.

[38] H. Gu, "Experimental observation of transition from chaotic bursting to chaotic spiking in a neural pacemaker," Chaos: An Interdisciplinary Journal of Nonlinear Science, vol. 23, no. 2, Article ID 023126, 2013.

[39] H. G. Gu, B. B. Pan, G. R. Chen, and L. X. Duan, "Biological experimental demonstration of bifurcations from bursting to spiking predicted by theoretical models," Nonlinear Dynamics, vol. 78, no. 1, pp. 391-407, 2014.

[40] B. Jia, H. Gu, and L. Xue, "A basic bifurcation structure from bursting to spiking of injured nerve fibers in a two-dimensional parameter space," Cognitive Neurodynamics, vol. 11, no. 2, pp. 189-200, 2017.

[41] Z. Zhao and H. Gu, "Transitions between classes of neuronal excitability and bifurcations induced by autapse," Scientific Reports, vol. 7, p. 6760, 2017.

[42] H. G. Gu and B. B. Pan, "A four-dimensional neuronal model to describe the complex nonlinear dynamics observed in the firing patterns of a sciatic nerve chronic constriction injury model," Nonlinear Dynamics, vol. 81, no. 4, pp. 2107-2126, 2015.

[43] H. Gu and B. Pan, "Identification of neural firing patterns, frequency and temporal coding mechanisms in individual aortic baroreceptors," Frontiers in Computational Neuroscience, vol. 9, no. AUGUST, article no. 108, 2015.

[44] Y. Wang, C. Wang, G. Ren, J. Tang, and W. Jin, "Energy dependence on modes of electric activities of neuron driven by multichannel signals," Nonlinear Dynamics, vol. 89, no. 3, pp. 19671987, 2017.

[45] L. Yang, W. Liu, M. Yi et al., "Vibrational resonance induced by transition of phase-locking modes in excitable systems," Physical Review E: Statistical, Nonlinear, and Soft Matter Physics, vol. 86, no. 1, Article ID 016209, 2012.

[46] L. Yang and Y. Jia, "Effects of patch temperature on spontaneous action potential train due to channel fluctuations: Coherence resonance," BioSystems, vol. 81, no. 3, pp. 267-280, 2005.

[47] B. C. Bao, Z. Liu, and J. P. Xu, "Steady periodic memristor oscillator with transient chaotic behaviours," IEEE Electronics Letters, vol. 46, no. 3, pp. 228-230, 2010.

[48] B. Muthuswamy, "Implementing memristor based chaotic circuits," International Journal of Bifurcation and Chaos, vol. 20, no. 5, pp. 1335-1350, 2010.

[49] G. Ren, Y. Xu, and C. Wang, "Synchronization behavior of coupled neuron circuits composed of memristors," Nonlinear Dynamics, vol. 88, no. 2, pp. 893-901, 2017.
[50] M. Lv and J. Ma, "Multiple modes of electrical activities in a new neuron model under electromagnetic radiation," Neurocomputing, vol. 205, pp. 375-381, 2016. 


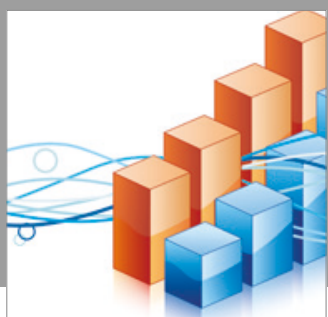

Advances in

Operations Research

vatersals

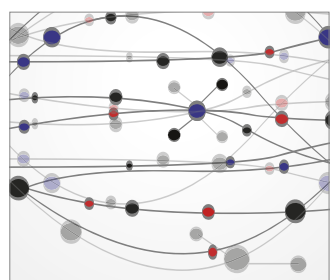

\section{The Scientific} World Journal
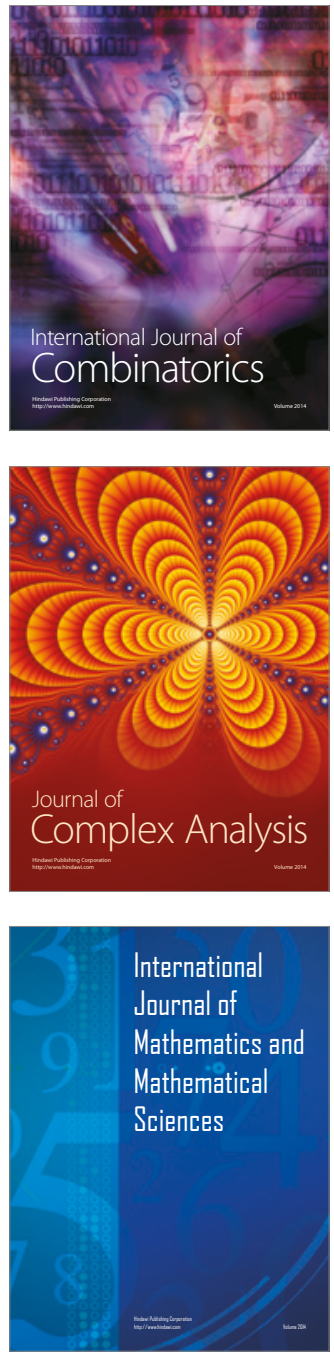
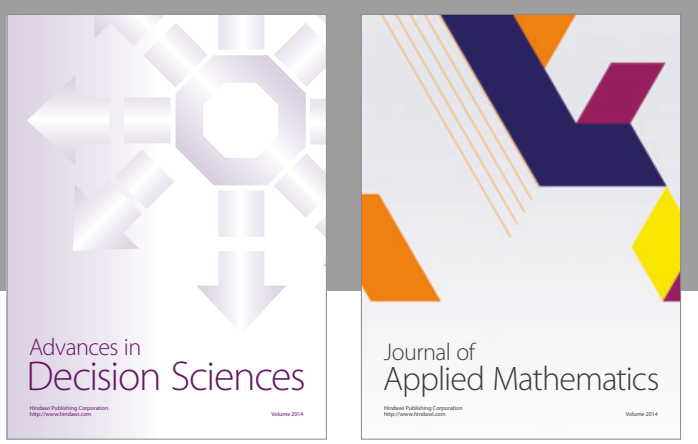

Algebra

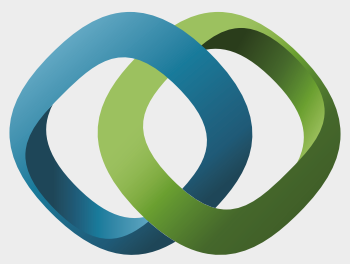

\section{Hindawi}

Submit your manuscripts at

https://www.hindawi.com
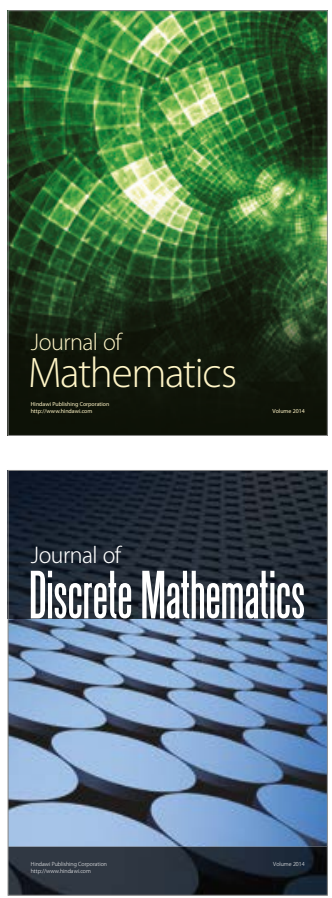

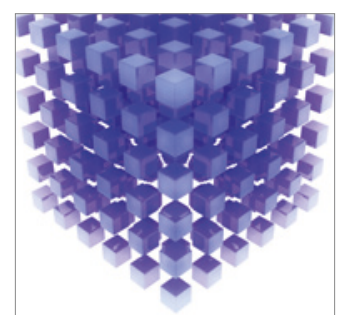

Mathematical Problems in Engineering
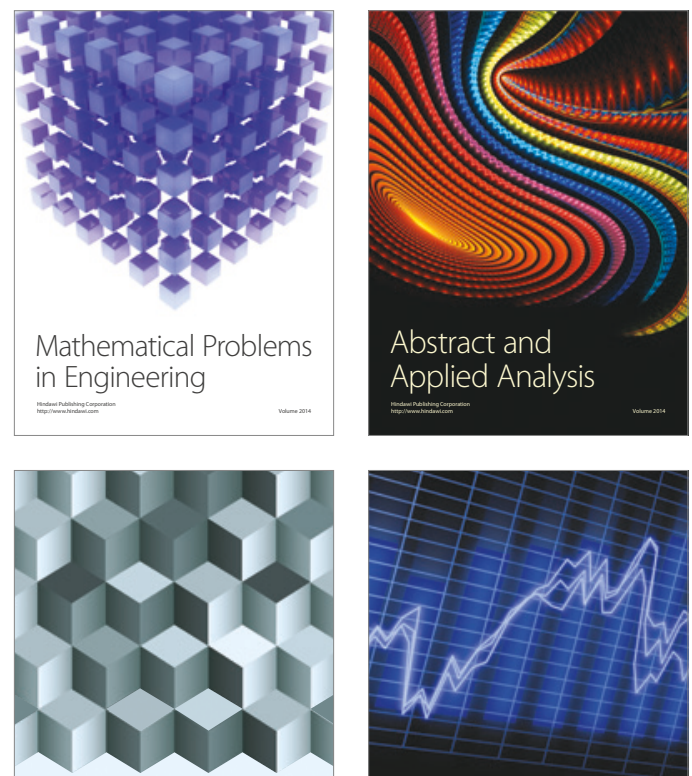

Journal of

Function Spaces

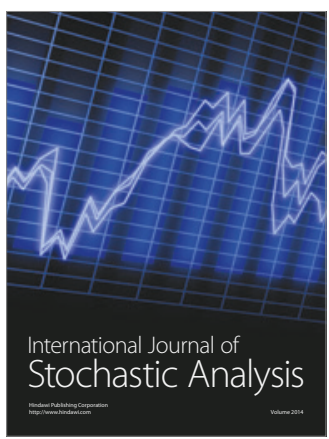

Probability and Statistics
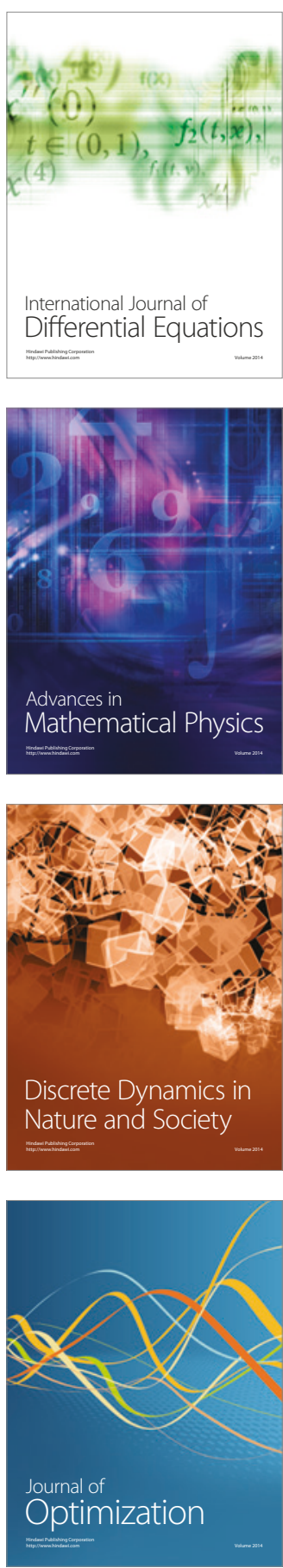